\title{
Intramedullary spinal cysticercosis: response to albendazole: case reports and review of literature
}

\author{
Ravindra Kr Garg and Devika Nag \\ Department of Neurology, King George's Medical College, Lucknow - 226003, India
}

Keywords: neurocysticercosis; spinal cord; albendazole; intramedullary cysticercosis; myelopathy

\section{Introduction}

In India cestode infection 'neurocysticercosis' is the commonest parasitic disease of the central nervous system; it may involve the brain parenchyma, the meninges or ventricles and, infrequently it involves the spinal cord. Involvement of spinal cord has variably been reported to be $1-5 \% .^{1,2}$ We report two cases of intramedullary spinal neurocysticercosis, which is a rare disease.

\section{Case reports}

Case I

An 11-year-old boy presented with a 2 days history of weakness of both lower limbs and inability to pass urine, for which he was catheterized. On examination there was 2/5 (MRC Scale) power in the lower limbs; the deep tendon reflexes were brisk in the lower limbs, and ankle clonus was present bilaterally. The plantar responses were extensor, and the cremastric reflexes were absent. There was impairment of sensation in both lower limbs from T10 dermatome level, all modalities of sensations being affected. Examination of the spine did not reveal any tenderness or deformity.

Serum biochemical, haematological parameters and urinalysis were normal. Plain X-rays of the thoracic spine and a myelogram were normal. Cerebrospinal fluid (CSF) examination revealed $68 \mathrm{mg} \%$ proteins, 16 cells $/ \mathrm{cumm}^{3}$ (all mononuclear), sugar $42 \mathrm{mg} \%$. Enzyme-linked immunosorbent assay (ELISA) examination of the CSF and serum were negative for tuberculosis, but were positive for cysticercosis. Magnetic resonance (MR) study of the spine showed a ring shaped, cysticercus lesion with an eccentric dot representing the scolex of larvae at the T9 level (Figure 1). Cranial computed tomography (CT) was normal. The patient was given albendazole $(15 \mathrm{mg} / \mathrm{kg}$ for 4 weeks) along with corticosteroids, and his condition improved considerably, so that he was able to walk independently 2 months after the albendazole therapy. Repeat MR study revealed disappearance of the lesion.

Correspondence: RK Garg, Deep Bhawan, behind Bank of Boroda, Bus Station, Sultan Pur 228001, India
Case II

A 10-year-old boy presented with asymmetrical weakness of both lower limbs of 4 days duration. The right lower limb was affected 2 days before the left. Along with involvement of left lower limb, he also developed hesitancy of urination. Tingling and numbness up to the knees were present from the onset of the illness. Whilst in hospital he had two left partial motor seizures. On examination, the patient had two pea-sized non-tender subcutaneous nodules over the flexor aspect of left forearm. Neurological examination revealed power in the right lower limb 2/5 (MRC scale), and in the left power was 3/5 (MRC scale). The legs were hypertonic. Both knee and ankle reflexes were brisk, and the plantar reflexes were extensor. The abdominal and cremastric reflexes were absent. Distal to T9 dermatome all modalities of sensations were impaired. Examination of the spine was normal.

The blood and urine biochemical parameters were normal. Biopsy of a subcutaneous nodule revealed cysticercus larva. Plain X-rays of the thoracic spine were normal. The CSF had $110 \mathrm{mg} \%$ proteins, 70 cells (all mononuclears), and $38 \mathrm{mg} \%$ sugar. ELISA for cysticercal antigen was negative in the CSF and serum. An MR study showed a ring shaped granulomatous lesion at T8 level (Figure 2). A cranial CT had showed a ring enhancing lesion in the right occipital region (Figure 3); the ring also had an eccentric dot. Albendazole therapy $(15 \mathrm{mg} / \mathrm{kg}$ for 4 weeks $)$ was given along with carbamazepine $(200 \mathrm{mg}$ thrice a day) and corticosteroids. There was no further seizure. Motor power improved and he was able to stand independently when he was discharged. He could not be followed up further.

\section{Literature review and discussion}

The larval form of pork intestinal tapeworm Taenia solium is the parasite responsible for neurocysticercosis. Humans are the definitive host for $T$. solium and usually harbour the adult tapeworm in the small intestine as an asymptomatic infection. Both humans and pigs are intermediate hosts for the embryonic form of $T$. solium and develop neurocysticercosis, and 
muscular cysticercosis after eating food contaminated with fertilized eggs excreted in the faeces of parasite carriers. Once in the intestine the eggs lose their sheath by the action of intestinal enzymes which liberate oncospheres (hexacanth embryo). These embryos penetrate the intestinal wall and enter the blood stream and lodge in various body tissues.

Classification and mode of spread to the spinal cord In the spinal cord the cysts are usually leptomeningeal and only rarely intramedullary, producing arachnoiditis or mass effects. Cysticercus cysts in the spinal subarachnoid space occur six to eight times more frequently than do intramedullary cysts. Leptomeningeal forms may be seen in association with cysticerci of the posterior cranial fossa and are best explained by downward migration of the cysts from the basal cisterns of the brain into the spinal canal through the cerebrospinal fluid pathways. ${ }^{1,4}$ Hematogenous spread is responsible for intramedullary spinal cysticercosis. Rarely, leptomeningeal lesions can also have a haematogenous origin. Transventricular migration of a cyst into the central canal of the spinal cord can also be responsible for the intramedullary spinal cysticercosis. Although the hypothesis of ventriculo-ependymal spread is unsubstantiated, this may offer a plausible explanation for the frequent occurrence of solitary intramedullary cysts. 5,6

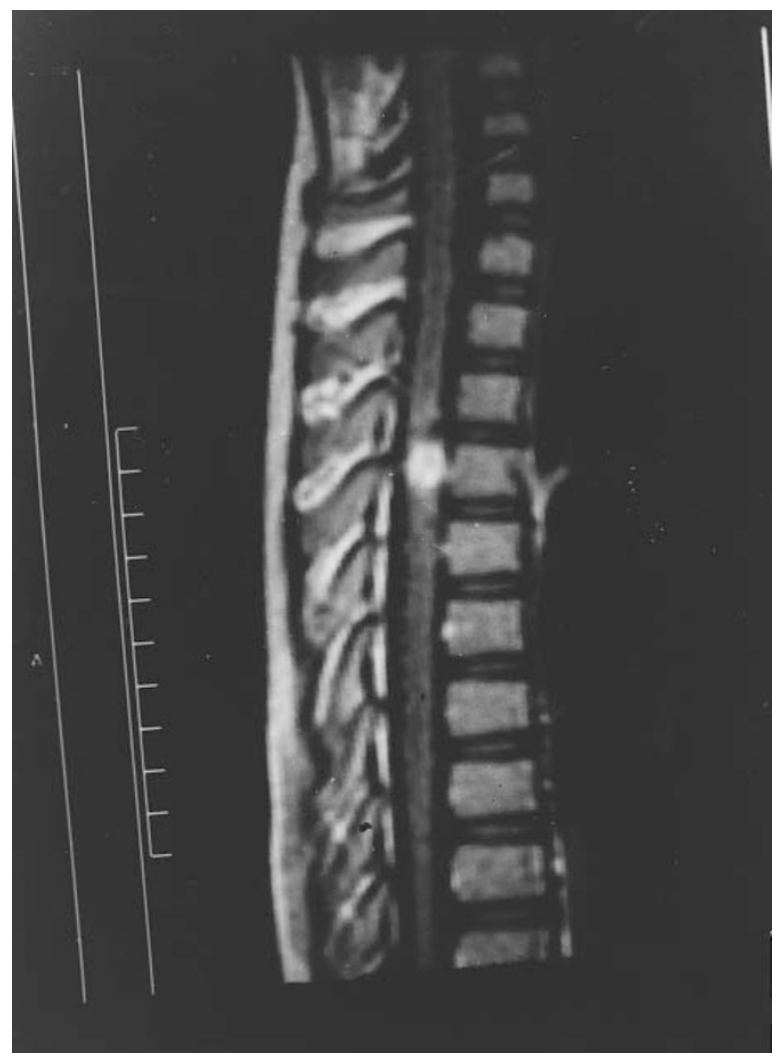

Figure 1 Sagittal $\mathrm{T}_{1}$-weighted $\mathrm{MR}$ image of the thoracic spine showing ring shaped hyperintensity with eccentric dot at T9

\section{Pathology and clinical manifestations}

Pathophysiologically there are three possible mechanisms by which neurological symptoms and signs might appear in patients with spinal cysticercosis: (1) an inflammatory reaction caused by the metabolites of the

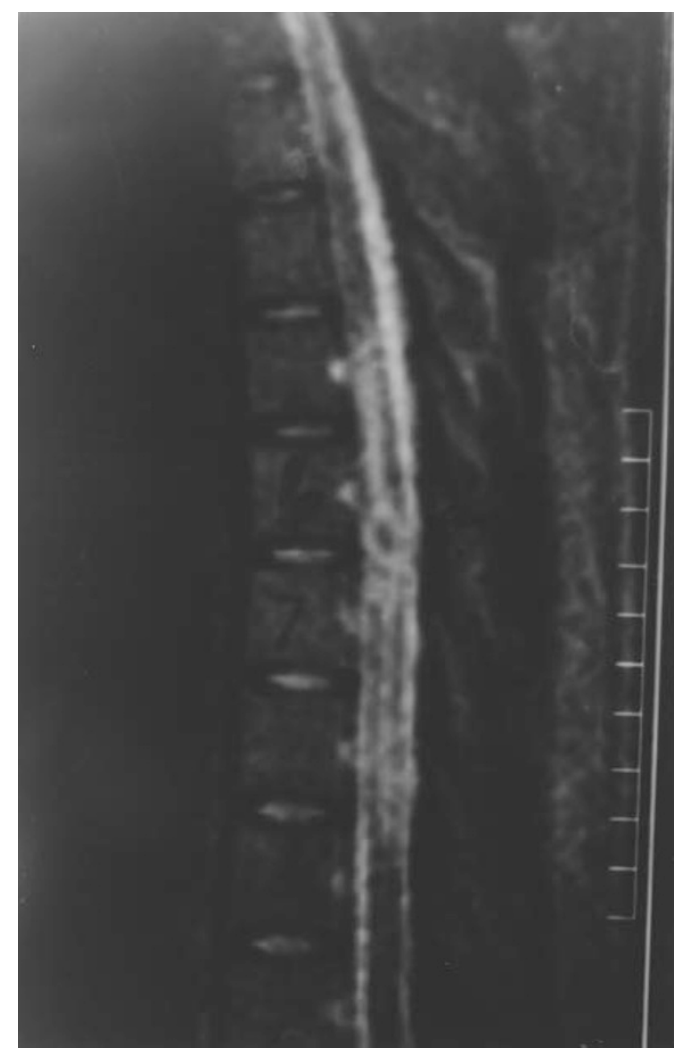

Figure 2 Saggital $T_{1}$-weighted $M R$ image of the thoracic spine demonstrating a hyperintense ring at $\mathrm{T} 8$. The lesion represents granulomatous stage of cysticercal lesion

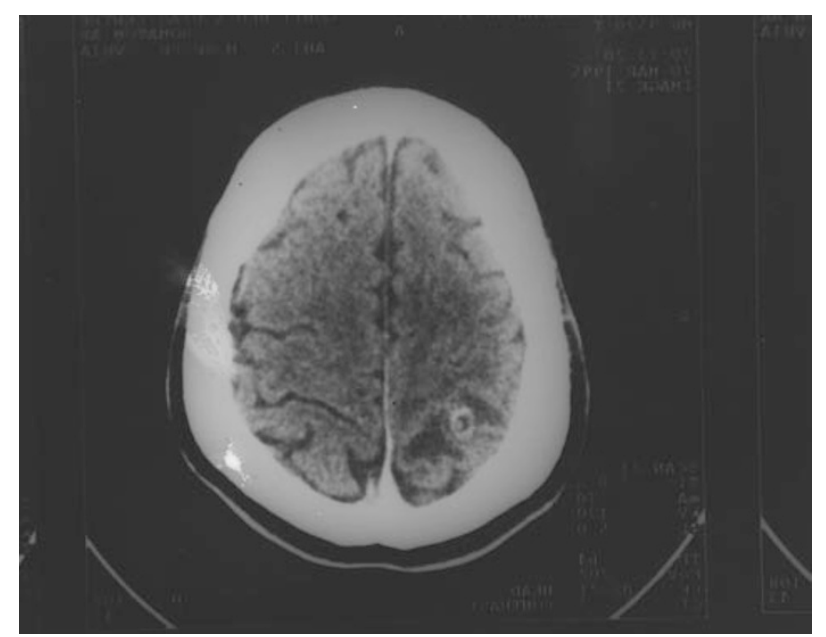

Figure 3 Contrast enhanced cranial computed tomography showing a ring enhancing lesion with eccentric dot in right occipital region 
parasite or degenerated larval remains; (2) the mass effect of intramedullary or extramedullary cysts (3) cord degeneration due to pachyleptomeningitis or vascular insufficiency. In any individual with spinal cysticercosis, all three of these mechanisms may play a part. Hydromyelic cavities may occur as a consequence of intramedullary cysts resulting in deficits over a larger area of involvement. ${ }^{1,7,8}$

The clinical manifestations of spinal neurocysticercosis are directly related to the location of the parasite. Intramedullary cysts cause motor weakness and a sensory level distal to the level of the lesion. Sphincter disturbances are a common feature in such patients. Leptomeningeal cysts produce a combination of root pain and weakness of subacute onset and progressive course. Intramedullary neurocysticercosis is more often found in the thoracic region of spinal cord, although it can develop in the lumbosacral region. 9,10 As haematogenous dissemination is proportional to the blood flow to the spinal cord, the thoracic cord which receives the greatest blood supply, is most often affected. ${ }^{11}$ Fifty percent of cases in the autopsy series published by Souza Queiroz et $a l^{5}$ were noted to have sites of involvement other than the spine. Of these $30 \%$ had intracerebral involvement and $25 \%$ had muscular involvement.

\section{Diagnosis}

Cerebrospinal fluid and serological tests

The cerebrospinal fluid findings typically include a moderate lymphocytic pleocytosis, a variable eosinophilic pleocytosis and an elevated protein level and a normal or low glucose level. Serological tests, eg enzyme-linked immunosorbent assay (ELISA) in the serum and the CSF, are very sensitive in establishing the diagnosis of neurocysticercosis.

\section{Imaging}

The radiographic appearance of extramedullary cysticercal cysts by myelography and postmyelography computed tomography are virtually indistinguishable from other cystic or nodular lesions of the spinal cord. Myelography may show multiple filling defects in the column of contrast medium. In intramedullary cysticercosis, myelography is frequently normal. Mobility of a cysticercus cyst on myelography is a unique feature of diagnostic significance. It is also important that one should be aware of the possibility that mobility of cyst may occur between the time of a myelographic study and surgery. ${ }^{12}$ The unattached mobile cysts in the spinal subarachnoid space probably remain asymptomatic since they are soft and small enough to pass through the subarachnoid space.

MR is currently the investigation of choice. It will not only show the cysts, the precise location and the proximity to neural structures, but also visualization of the scolex of cysticercosis larvae. ${ }^{10}$ The signal properties of the spinal cysticercus cysts are similar to those described in parenchymal brain cysts. At times contrast enhancement with gadopenteate dimeglumine administration is required for enhancement of the scolex and can help in establishing the proper diagnosis. $9,13,14$

\section{Treatment}

There are no controlled studies regarding the efficacy of medical therapy for spinal cysticercosis because of the rarity of the disease. The idea of medical therapy is presently based on the reported efficacy of anticysticercal drugs for cerebral cysticercosis and in isolated cases for spinal cysticercosis. Both praziquantel and albendazole have been found effective in both forms of spinal cysticercosis. ${ }^{15,16}$ Current therapy for the leptomeningeal form of spinal cysticercosis is laminectomy and surgical resection, and if cerebrospinal fluid findings are suggestive of active arachnoiditis a course of corticosteroids may be administered. ${ }^{12}$ Intramedullary spinal cysts are usually surgically resected for confirmation of the diagnosis, however, therapy with anticysticercal drugs may be tried when the diagnosis is confirmed or is suspected before surgery. ${ }^{10}$ Concomitant corticosteroids must be given along with anticysticercal treatment to avoid further damage of the spinal cord due to subsequent inflammatory reaction in the cyst wall.

\section{Comments and conclusion}

Our two patients illustrate similar morphological pictures of intramedullary spinal cysticercal lesions as seen in cerebral cysticercosis. These lesions represent the granulomatous stage of the disease. One of our patients also had a similar lesion in the brain. Both patients presented with an acute transverse cord syndrome. In the past, compression of spinal cord or nerve roots by the parasite was treated by surgical decompression. The efficacy of anticysticercal drugs can alter the indications for surgery in spinal neurocysticercosis. Several reports have appeared indicating cure of spinal subarachnoid involvement and an excellent response in intramedullary cysticercosis with albendazole. In the absence of data from controlled studies the treatment of spinal cysticercosis with an anticysticercal drug should be considered empirical. Response to this form of treatment is not universal. Banders and colleagues ${ }^{17}$ noted that extraparenchymal disease may be difficult to control and several courses of medical therapy may be required.

\section{References}

1 Akiguchi I et al. Intramedullary spinal cysticercosis. Neurology 1979; 29: $1531-1534$.

2 Webbe G. Human cysticercosis: Parasitology, pathology, clinical manifestations and available treatment. Pharmacol Ther 1994; 64: $175-200$. 
3 Del Brutto OH, Sotelo J, Roman GC. Therapy for neurocysticercosis: A reappraisal. Clin Infect Dis 1993; 17: 730-735.

4 Isidro-Llorens A, Dachs F, Vidal J, Sarrias M. Spinal cysticercosis: Case report and review. Paraplegia 1993; 31: $128-$ 130.

5 Souza-Queiroz L, Pellegrini A, Callegaro D, Lopez de Faria L. Intramedullary cysticercosis. J Neurol Sci 1975; 26: 61 - 70 .

6 Kishore LT et al. Intramedullary spinal cord cysticercosis. A case report and literature review. Indian J Pathol Microbiol 1991; 34: $219-221$.

7 Kahn P. Cysticercosis of the central nervous system with amyotrophic lateral sclerosis: Case report and review of literature. J Neurol Neurosurg Psychiatry 1972; 35: 81 - 87.

8 Gupta PK et al. Spinal subarachnoid cysticercosis. Neurol India 1995; 43: $60-61$.

9 Castillo M, Quencer RM, Post MJD. MR of intramedullary spinal cysticercosis. AJNR 1988; 9: $393-395$.

10 Agrawal V, Thomas M, Maheshwari MC. Intramedullary cysticerci. J Assoc Physicians India 1995; 43: 138.
11 Sharma BS, Banerjee AK, Kak VK. Intramedullary spinal cysticercosis. Clin Neurol Neurosurg 1987; 82: $2111-2116$.

12 Kim KS, Weinberg PE. Spinal cysticercosis. Surg Neurol 1985; 24: $80-82$.

13 Kochan JP, Quencer RM. Imaging of cystic and cavitory lesions of the spinal cord and canal: The value of MR and intraoperative sonography. Neuroimaging Clin North Am 1991; 29: 867-911.

14 Zee C-S et al. MR imaging of neurocysticercosis. J Compt Assist Tomogr 1988; 12: 927 -934.

15 Del Brutto OH, Sotelo J. Neurocysticercosis. An update. Rev Infect Dis 1988; 10: $1075-1087$.

16 Del Brutto OH. Diagnosis and management of cysticercosis. $J$ Trop Geogr Neurol 1992; 2: 1 -9.

17 Bandres JC et al. Extraparenchymal neurocysticercosis: Report of five cases and a review of management. Clin Infect Dis 1992; 15: $799-803$. 